\title{
Généralités sur les thermoclines, leur genèse et leur prévision
}

\section{Exposé introductif}

\author{
par H. Lacombe \\ Professeur d'Océanographie Physique \\ au Muséum de Paris
}

L'objectif de cette deuxième journée de la session de la S.H.F. est l'étude de la perturbation des écoulements océaniques par les gradients de température. Peut-être est-il utile de rappeler quelques généralités sur la structure thermique verticale marine, ses caractères et sa genèse.

\section{I - Allure générale de la structure thermique marine sur la verticale}

a) Cette structure se présente en général comme la superposition de trois couches:

- Une couche superficielle d'une épaisseur de 50 à $200 \mathrm{~m}$, caractérisée par le fait qu'en général la température y est voisine de celle que l'on trouve en surface. Toutefois, dans les 15 à $40 \mathrm{~m}$ superficiels, dans les latitudes tempérées ou élevées, l'effet de l'échauffement saisonnier se fait sentir (voir ci-dessous: thermocline saisonnière).

- Au-dessous, dans une couche s'étendant sous la couche superficielle et jusqu'à un millier de mètres, en général, on rencontre une notable diminution de la température à immersion croissante: c'est la thermocline principale ou permanente (car elle varie fort peu en cours d'année).

- Au-delà, le gradient thermique tend généralement vers zéro: ce sont les eaux profondes (fig. 1).

$$
\text { ; }
$$

Dans certaines aires, notamment dans les régions polaires et, également, dans certaines mers comme la Méditerranée et la mer Rouge, l'eau est à peu près homotherme sous la thermocline saisonnière.

\section{$*$}

La définition des limites supérieure et inférieure de la thermocline est souvent très floue, particulièrement entre le bas de la thermocline principale et la couche profonde.

b) Mais aux niveaux proches de la surface (0-50 m par ex.), la distribution thermique présente de grandes variations: les unes saisonnières; les autres avec des périodicités beaucoup plus courtes, par ex. diurnes.

Là, en effet, les eaux sont sujettes à évoluer rapidement en température car les effets de l'absorption du rayonnement solaire (échauffement) et des pertes vers le haut s'y font sentir; en outre, ces couches sont sujettes au brassage mécanique lié aux vents (vagues et courant). Il convient de décrire les effets des transferts thermiques à travers la surface marine et ceux du brassage mécanique.

Le bilan thermique d'une colonne d'eau, de section droite unité, dans le cas où on néglige la chaleur transportée par les courants (advection), est la résultante:

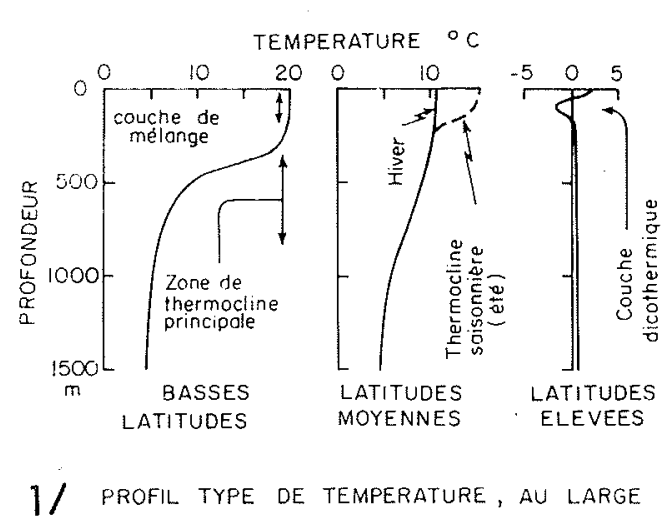




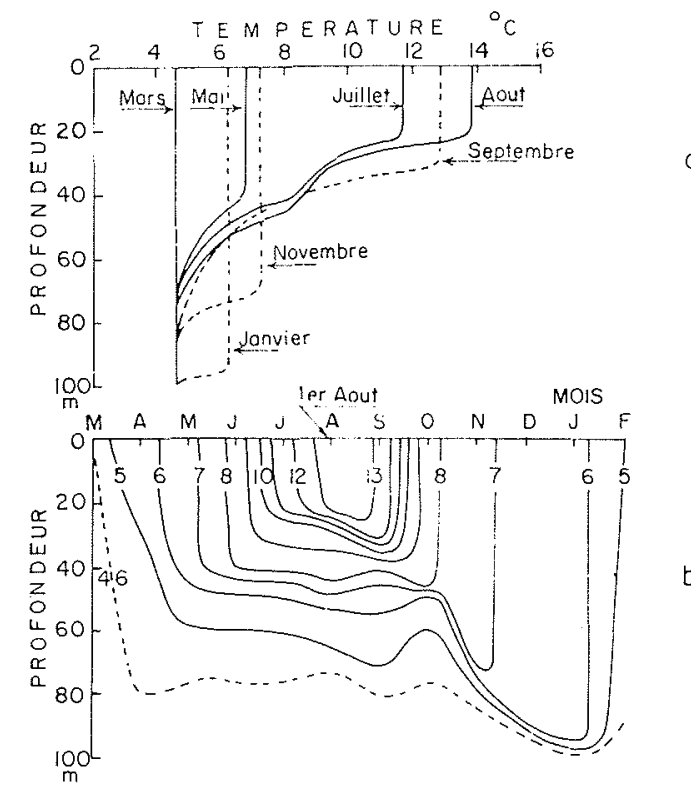

$2 /$ CROISSANCE ET DECROISSANCE DE LA THERMOCLINE
SAISONNIERE AU $50^{\circ} \mathrm{N}, 145^{\circ} \mathrm{W}$ DANS LE NORD EST
DE L'OCEAN PACIFIQUE (groupe ocḱnogrophique du
pocifique, Conodo)

- des gains thermiques dus à l'absorption du rayonnement solaire dans les couches marines proches de la surface;

- de pertes dues au rayonnement infra-rouge de la surface, de la chaleur latente de l'eau évaporée, empruntée à la mer, de la chaleur sensible cédée par l'eau à l'air (si $T_{\text {eau }}>T_{\text {air }}$ ).

Les gains sont fonctions de l'heure, de la latitude et de la saison (déclinaison du soleil). La moyenne journalière (ou hebdomadaire) de ces facteurs dépend relativement peu de la saison dans les basses latitudes (la hauteur du soleil à midi varie peu au cours de l'année), mais elle en dépend notablement dans les latitudes moyennes et plus encore dans les latitudes élevées.

Quant à l'effet du brassage mécanique des vagues et du vent, qui est intense au moment des coups de vent, il se produit en tous lieux à une échelle de temps de l'ordre de la semaine.

Il résulte de ces faits que:

$1^{\circ}$ Dans les basses latitudes, la structure thermique des couches superficielles varie peu au cours de lannée: quelle que soit la saison, la température est peu variable jusqu'à $50-100 \mathrm{~m}$ et est proche de la température de surface. Auldessous se trouve la thermocline permanente.

$2^{\circ}$ Dans les latitudes tempérées ou élevées, l'excès notable, pendant l'été, de l'échauffement solaire sur les pertes, a pour effet de susciter alors une thermocline saisonnière sous une "couche de mélange » à peu près homotherme, résultant d'une « redistribution » de la chaleur emmagasinée, sous l'effet de brassages mécaniques divers, cette couche a de 15 à $40 \mathrm{~m}$ d'épaisseur en général. Cette thermocline naît au printemps (mai : hémisphère Nord), s'accentue en été et disparaît à la fin de l'automne. L'écart thermique de part et d'autre de la thermocline est plus élevé dans les latitudes tempérées que dans les hautes latitudes (fig. 2).

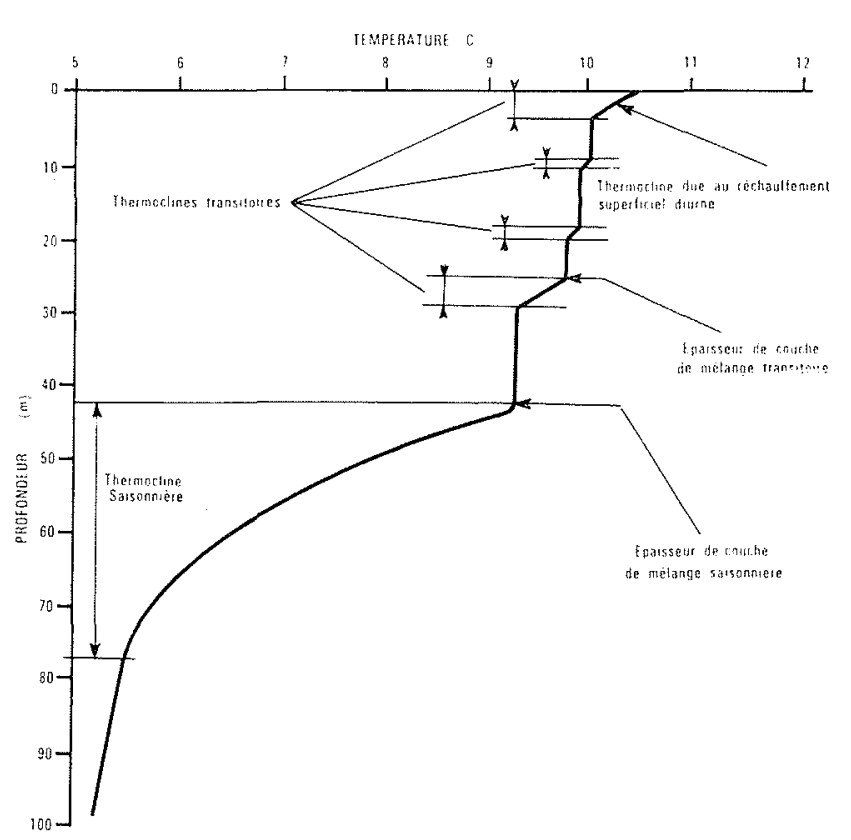

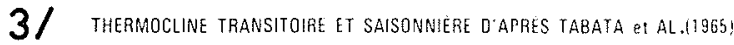

$3^{\circ}$ Au cours de l'été, par périodes de calme entre les coups de vent, des thermoclines diurnes ou transitoires sont suscitées à des niveaux peu profonds, du fait de la variation du rayonnement solaire au cours de la journée ou sous l'effet d'une redistribution thermique par vent faible ou moyen, dont l'effet de brassage n'atteint pas, en immersion, la thermocline saisonnière (fig. 3). La thermocline diurne par calme plat se trouve à quelques décimètries seulement.

\section{II - Les moteurs de la genèse des thermoclines}

a) La thermocline permanente. Sauf zones particulières (comme les ombilics chauds de la mer Rouge), le rayonnement solaire est la seule source pratique de chaleur pour la mer. Au haut de la thermocline permanente il ne parvient pratiquement pas. On est donc conduit à penser que le moteur prépondérant de la genèse de cette thermocline permanente est l'advection de chaleur par les courants marins et, notamment, dans les zones de «convergence» comme la mer des Sargasses: là convergent, dans la zone de l'anticyclone subtropical, des eaux superficielles entraînées au nord par les vents d'ouest, au sud par les alizés. II n'y a pas de «modèle» de genèse de la thermocline permanente qui soit effectivement mis en pratique.

b) Les autres thermoclines - saisonnière, diurnes et transitoires - sont principalement engendrées par des transferts verticaux d'énergie thermique et mécanique à travers la surface marine. Si l'advection joure un rôle, elle peut, dans la mesure où elle est connue, être prise en compte. Au large, on la considère généralement comme 
négligeable, à titre plus ou moins juste. Il en résulte que les «modèles» de thermocline usités prennent seulement en compte les transferts verticaux. Comme il semble que l'échelle des variations horizontales des causes de ceux-ci est très grande vis-à-vis de l'épaisseur de la couche de mélange, les «modèles» sont à une dimension $(z$, immersion). On postule aussi l'absence d'ondes internes.

\section{III - Les principes de base des modèles des thermoclines saisonnières, diurnes et transitoires}

\author{
$1^{\circ}$ Les facteurs déterminants en sont:
}

a) les transferts radiatifs et de chaleur à travers la surface;

b) les transferts d'énergie mécanique et les brassages qui en résultent.

\section{a) LES TRANSFERTS RADIATIFS ET THERMIQUES.}

Gain : L'absorption du rayonnement solaire est caractérisé par un coefficient $k$ global (c'est-à-dire pour l'ensemble des longueurs d'onde présentes), tel que la diminution $d l$ sur la verticale d'un flux lumineux $I(z)$, pour un accroissement $d z(\mathrm{~cm})$ d'immersion, est donnée, pour l'unité de temps, par :

$$
d I=-k(z) I(z) d z \quad k \text { en } \mathrm{cm}^{-1}
$$
de :

Cette absorption $d I$ entraîne un gain thermique unitaire

$$
d T=k(z) I d z
$$

car masse volumique et chaleur spécifique de l'eau sont voisines de 1 .

\section{Pertes:}

- rayonnement infra-rouge; assez difficile à évaluer, de l'ordre de $0,09 \mathrm{cal} / \mathrm{cm}^{2} . \mathrm{mn}$; des formules empiriques le donnent;

- évaporation de la mer $e(\mathrm{~cm})$ et perte $L e(L=$ chaleur de vaporisation de l'eau, environ $585 \mathrm{cal} / \mathrm{g}$ ); l'évaporation est fort mal connue encore, malgré diverses formules empiriques;

- pertes de chaleur sensible, mal connues aussi mais beaucoup plus faibles que les précédentes.

On néglige la production de chaleur par dissipation visqueuse.

La température marine, vers 100 ou $150 \mathrm{~m}$ de profondeur, est supposée invariable.

Les composantes du bilan thermique doivent, bien sûr, être connues avant toute prédiction de thermocline et il y a là, toujours, grande indécision.

\section{b) Les transferts D'Énergie MÉCANIQUe a Travers La SURFACE ET LEURS EFFETS.}

Ces transferts produisent, dans la couche superficielle, une augmentation de l'énergie cinétique turbulente, qui sert à fournir l'énergie potentielle nécessaire pour mélanger des couches en stratification stable.
L'énergie cinétique turbulente dans l'eau varie:

- du fait de la production d'énergie turbulente par les tensions de Reynolds agissant sur le courant moyen;

- du fait de la divergence sur la verticale du transfert vertical d'énergie mécanique turbulente produite en surface par les vagues (énergie cinétique et travail des fluctuations de pression);

- du fait de la dissipation;

- du fait de l'utilisation, d'une part, de l'énergie à travailler contre les forces de flottabilité dues à la stratification initialement stable.

$2^{\circ}$ Le modèle de K. L. Denman (1973) dérive de celui de Kraus et Turner (1967).

On écrit à la fois la conservation de l'énergie thermique et celle de l'énergie mécanique. Le modèle est complexe et conduit à deux équations différentielles simultanées où interviennent, d'une part la variation de la température de la couche mélangée, d'autre part la variation d'épaisseur de celle-ci. On y introduit le bilan total du rayonnement solaire et des pertes thermiques, les divers éléments de transfert, de production et d'utilisation de l'énergie mécanique turbulente. L'évaluation de celle-ci, en fonction de l'énergie cédée par le frottement tangentiel du vent sur la mer, comporte un facteur empirique $m$, dont la valeur est controversée.

Mais le modèle, qui fait agir simultanément les effets thermiques et dynamiques, permet une prédiction qui semble correspondre assez bien à la réalité pour les thermoclines diurnes et transitoires (fig. 4), bien que le coefficient $k$ d'absorption soit pris constant. Ceci nous paraît conduire à des oscillations thermiques diurnes, par temps calme, insuffisantes dans les couches très proches de la surface. Mais Saint-Marcoux introduit actuellement, dans le modèle, l'hypothèse $k$ variable avec $z$ (loi hyperbolique).

$3^{\circ}$ Nous avons proposé (1972-1973) un modèle beaucoup plus simple de thermocline (saisonnière et transitoires), qui sépare les effets thermiques des effets du brassage mécanique dû au seul vent. Mais on y postule cependant, dans les effets thermiques, des mouvements de convection provoquant l'homothermie de la couche de mélange: en effet, l'échauffement dû à l'absorption du rayonnement solaire se fait sentir dans une couche superficielle d'une épaisseur de l'ordre du décamètre; au contraire, le refroidissement est strictement «pelliculaire»; comme les énergies mises en jeu par ces deux phénomènes inverses sont comparables, il s'ensuit que la pellicule de surface tend à augmenter de densité, d'où des mouvements de convection établissant l'homothermie de la couche de mélange.

Curieusement, ce modèle très simple, assorti de l'hypothèse d'un coefficient d'absorption constant, conduit, pour la thermocline saisonnière, à une évolution de mois en mois de la situation thermique qui rappelle la réalité, sur la base de la connaissance simultanée des gains thermiques et des pertes thermiques (le bilan net ne suffit pas). Ainsi, on trouve (fig. 5,6) un minimum d'épaisseur de la couche de mélange en juin (hémisphère Nord) quand l'échauffement est maximal par rapport au refroidissement; une lente augmentation de l'épaisseur de la couche pendant l'été, avec une élévation thermique notable; puis, à partir de septembre, un épaississement de la couche de mélange et une diminution thermique; on peut comparer à la réalité marine (fig. 7), il semble donc que, pour le cas de la 


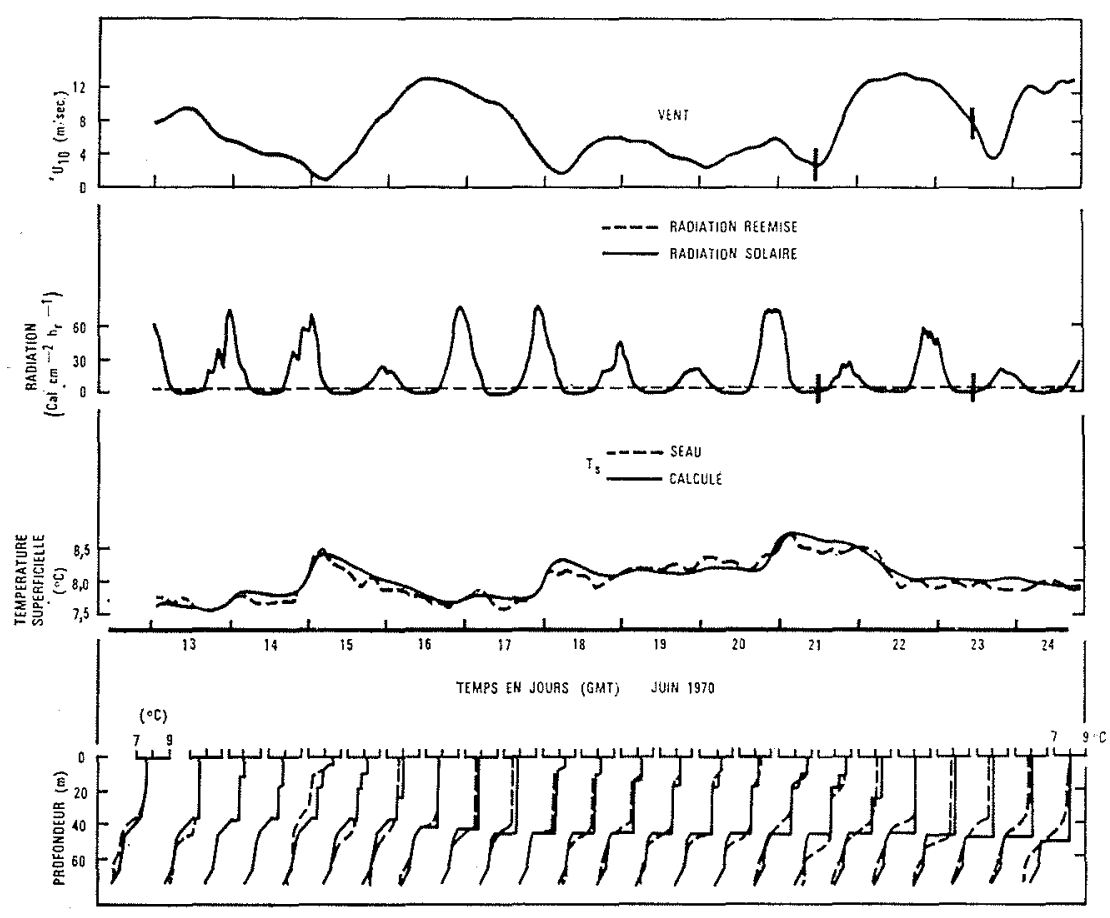

$4 /$ PERIOOE DU 13 aU 24 JUIN 1970. COMPARAISON ENTRE LES MESUAES (TRAITS POINTILLÉS) ET LES CALCULS (TRAITS PLEINS). D'APRES DENMAN

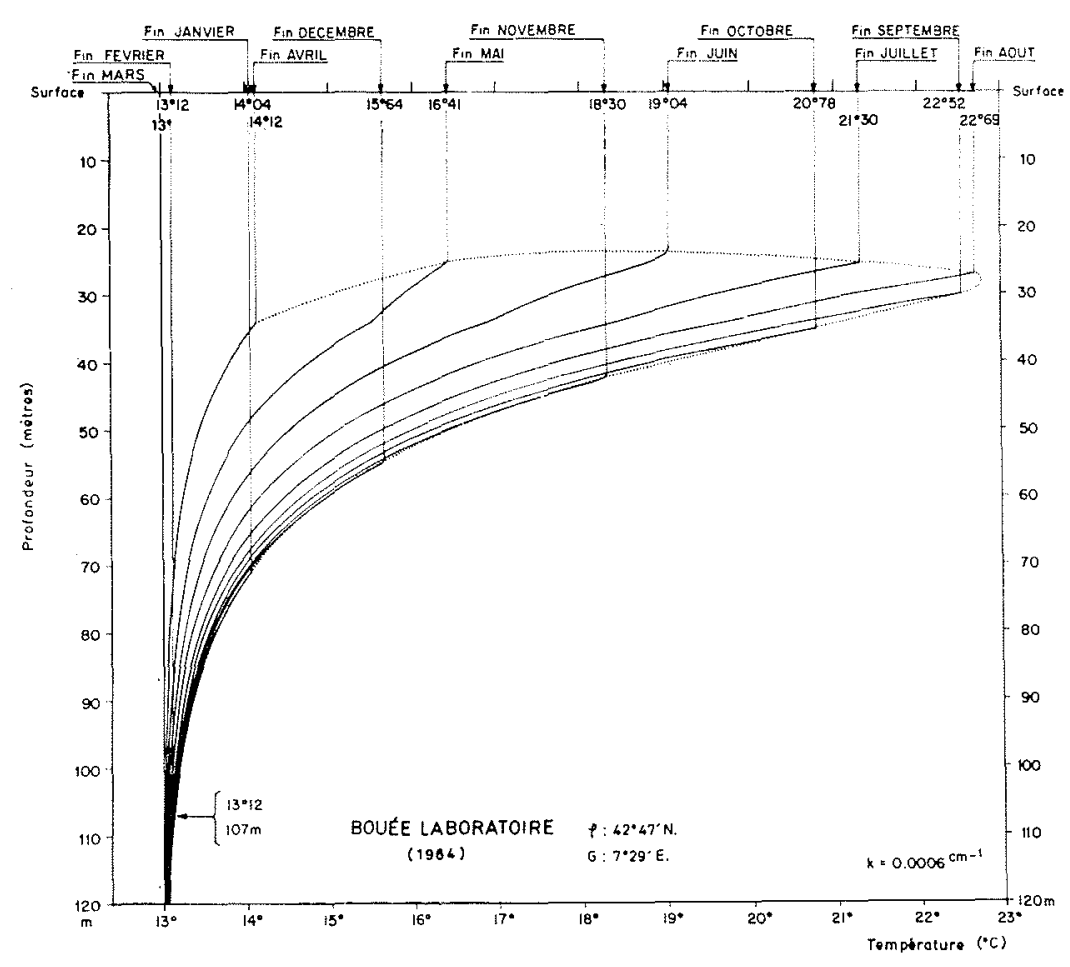

5 / evolution de la ihermocline saisonniere a la bouee laboratoire 


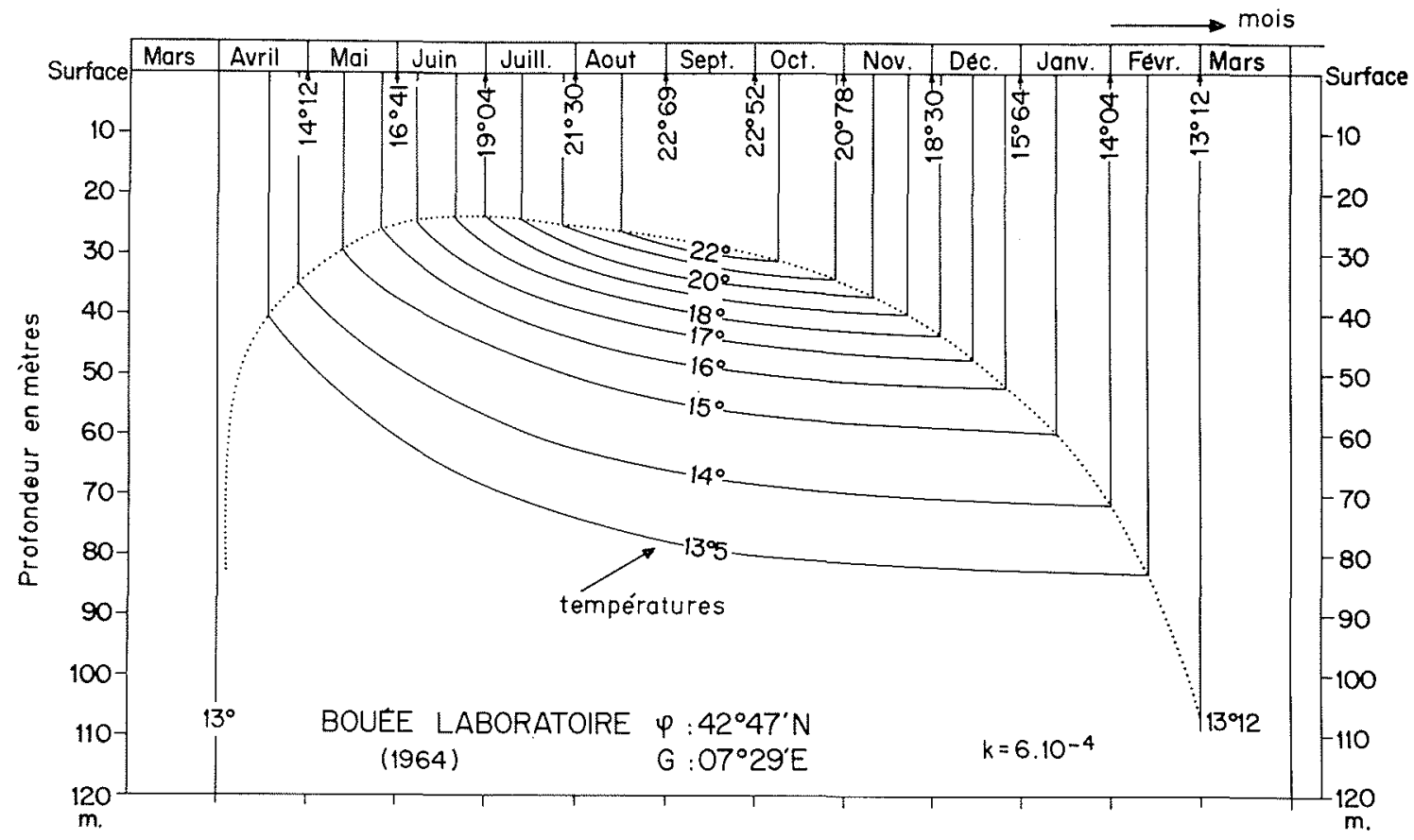

\section{1}

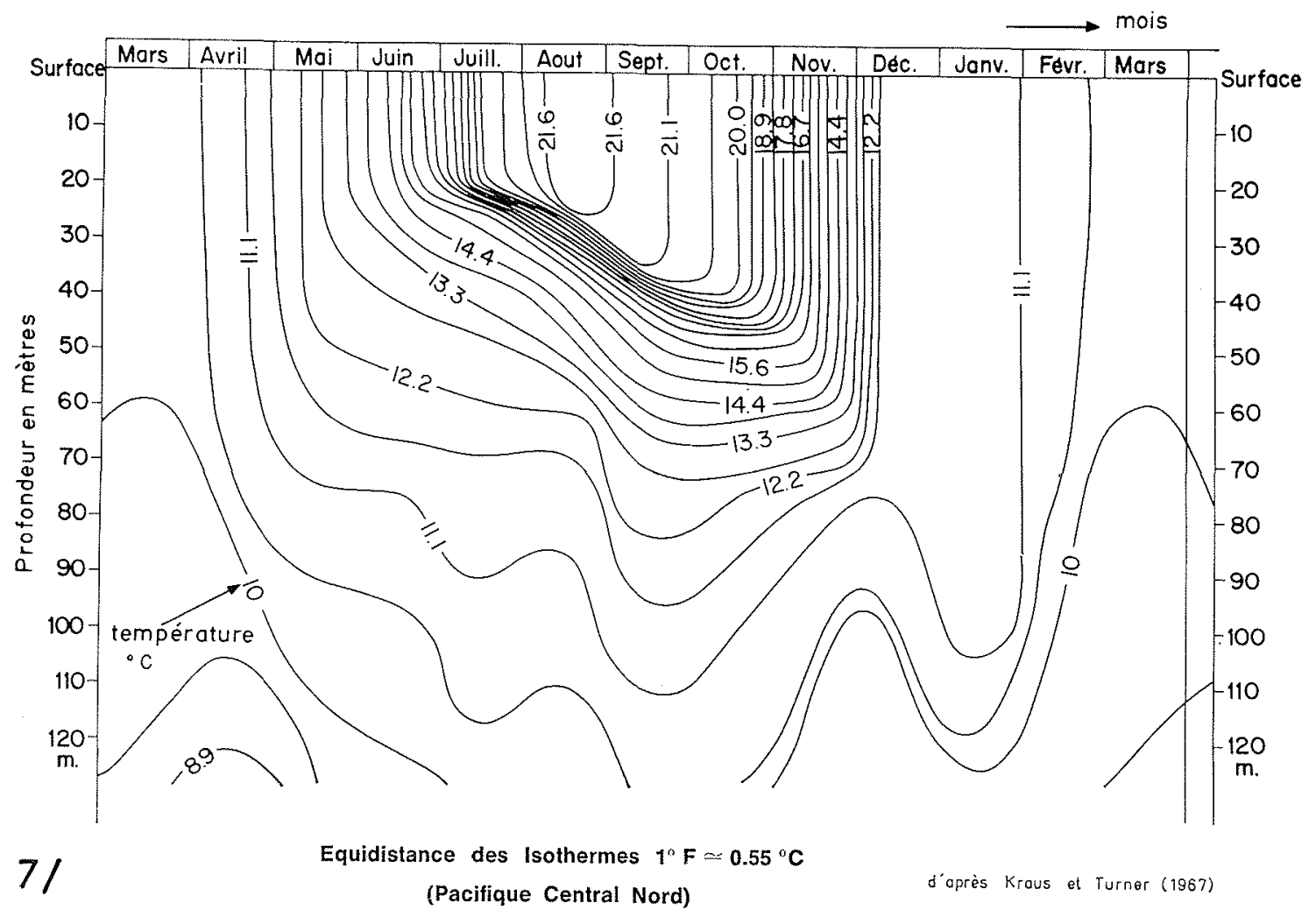




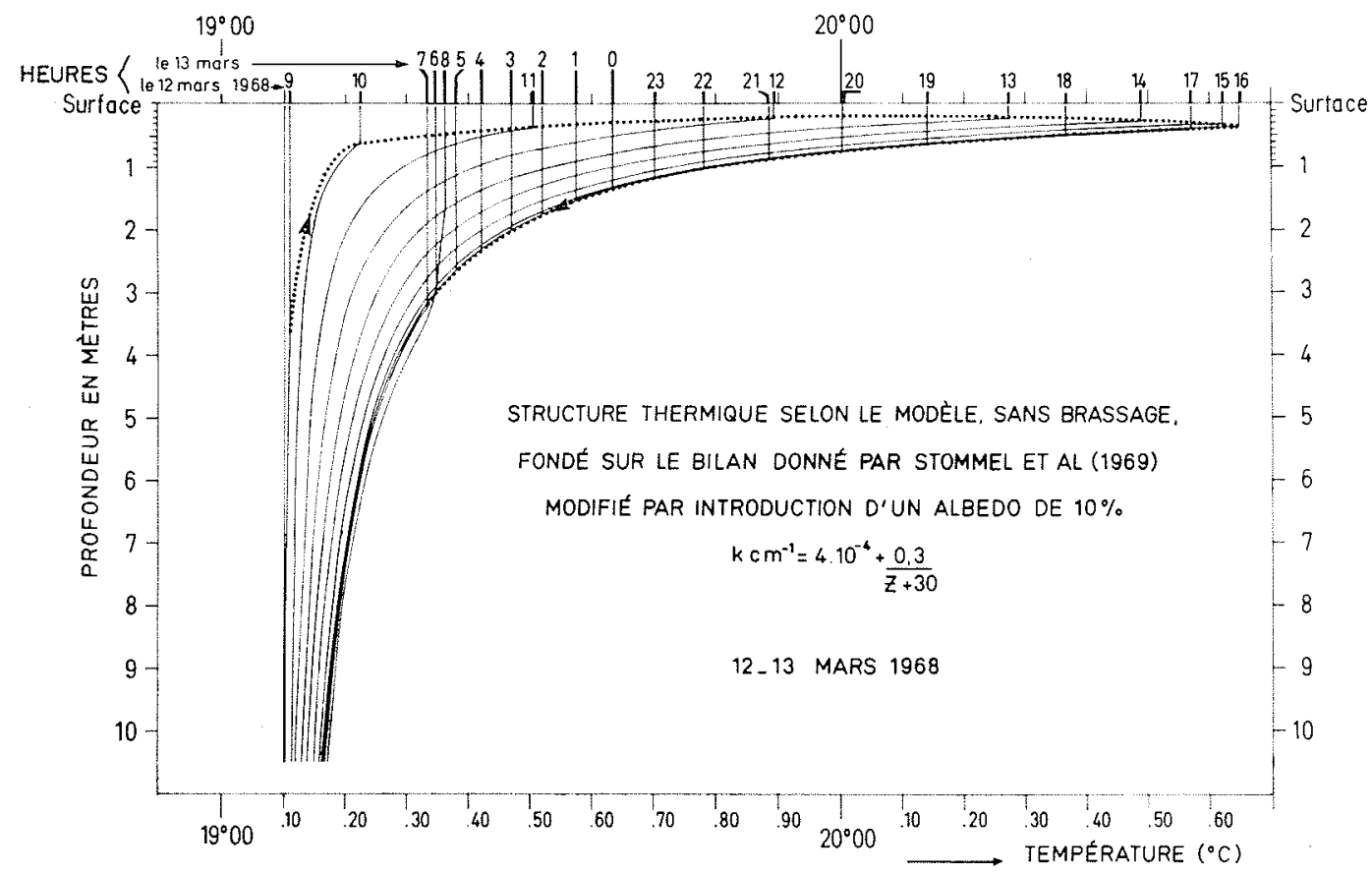

\section{$8 /$}

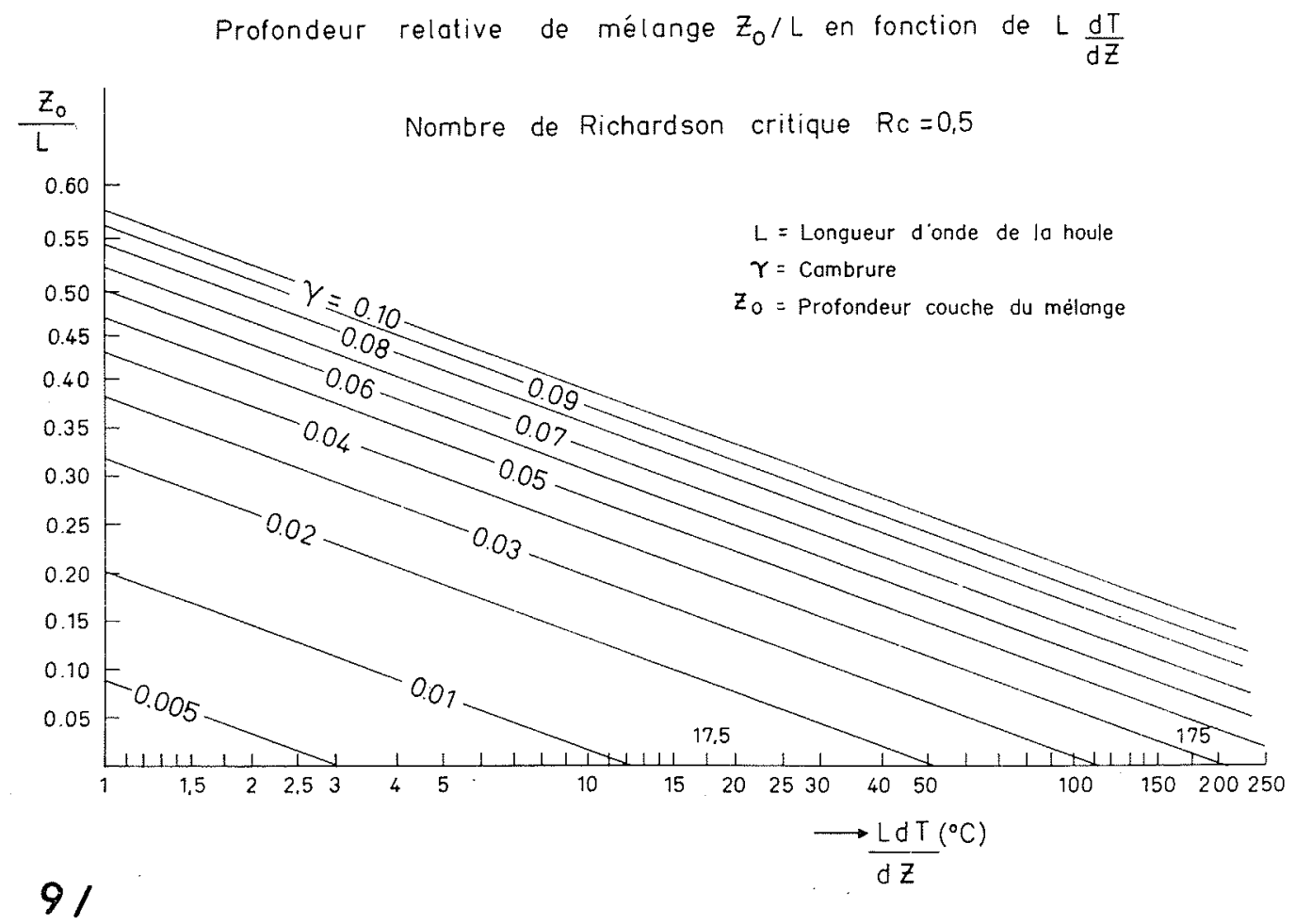


thermocline saisonnière, l'hypothèse $k=\mathrm{C}^{\text {te }}$ reproduise l'effet de brassage du vent.

$\mathrm{Si}$, en l'absence de vent, par temps calme, on prend une loi $k(z)$ hyperbolique en $z\left[k=k_{0}+\alpha /(z+a)\right]$ rappelant la réalité, le modèle rend compte de la formation de la thermocline diurne (fig. 8).

Lorsque le vent se lève, celui-ci, par agitation, suscite une redistribution thermique. Nous avons proposé, un peu intuitivement, que c'est l'agitation des vagues qui est principalement responsable de ce brassage; un nombre de Richardson est alors introduit, en partant du gradient de densité donné par le modèle sans vent et fondé sur le gradient du module des vitesses orbitales des particules dues aux vagues simultanément présentes. La valeur critique du nombre en question $(0,5)$ est déduite de l'observation d'un cas pratique, malheureusement unique, rencontré sur la bouée-laboratoire en 1969. On en déduit (fig. 9) l'épaisseur de la couche de mélange en fonction du gradient thermique initial et des caractères des vagues.
秋

J'espère que cette courte introduction n'était pas inutile pour rappeler, qualitativement au moins, les facteurs qui déterminent la structure thermique des couches marines superficielles.

\section{Bibliographie sommaire}

Denman (K. L.). - A time dependent model of the upper ocean. Journ. Phys. Oceantogr., vol. 3, $\mathrm{n}^{\circ}$ 2, April 1973, pp. 173-189.

KrAUS (G. B.), TURNER (J.S.). - A one-dimensional model of the seasonal thermocline. Tellus, 19, 1967, pp. 88-105.

LACombe (H.). - Sur un modèle simple de la thermocline saisonnière et sur sa prévision. C.R. Acad. Sc., D, 275, 1972, pp. 22112214.

Lacombe (H.). - Modèles simples de prévision de l'état thermique de la mer et de l'immersion de la thermocline. Amn. Hydr et Océanogr. (solis presse).

\section{Discussion}

Président : M. J. DUPORT

M. le Président remercie vivement $M$. le Professeur Lacombe pou son exposé, qui remet très clairement en mémoire le mécanisme des divers phénomènes qui seront étudiés au cours de cette deuxième journée de la Session.

Il ouvre ensuite la discussion en posant la question suivante :

Parmi les facteurs d'échange entre l'atmosphère et la surface liquide, l'action d'une pluie tombant sur la mer n'a pas été mentionnée cette action a-t-elle été déjà étudiée?

Il est certain, répond M. le Professeur Lacombe, que l'effet des précipitations sur la structure thermique peut être important d'une part, par son influence sur la densité, d'autre part, du fait que la température de la pluie est différente de celle de la mer. Le modèle de thermodine présenté ici n'en tient pas compte. Dan la réalité marine, on a pu suivre le processus de mélange par des mesures faites dans l'Atlantique.

L'introduction des effets de la salinité (augmentation due à l'évaporation) dans le modèle n'est probablement pas très difficile. Un calcul simple montre que l'effet sur la densité de l'augmentation de salinité est seulement de l'ordre de 25 à $30 \%$ de l'effet de la diminution de température, liée à l'évaporation.
M. CRÉPON apporte son expérience de nageur à bord de la «bouéelaboratoire »:

Vers 3 heures de l'après-midi, en plein mois de juillet, dit-il, on sent très fortement l'existence de la thermocline journalière aux abords de la bouée; si l'on fait la «planche» et si on laisse tomber ses bras verticalement, on sent l'eau très froide. Malheureusement, il s'avère difficile de faire des mesures de température tout près de la «bouée-laboratoire » car de petits courants permanents, existant dans son voisinage, créent une zone perturbée dans un rayon de 5 ou $6 \mathrm{~m}$ autour de celle-ci.

En effet, confirme M. le Professeur LACOMBE; aussi en 1969 , avions-nous installé, à 200 ou $300 \mathrm{~m}$ de la «bouée-laboratoire » une bouée satellite qui portait des capteurs thermiques à $10 \mathrm{~cm}, 50 \mathrm{~cm}$, $1 \mathrm{~m}, 2 \mathrm{~m}, 3 \mathrm{~m}$ et $5 \mathrm{~m}$ de la surface; c'est sur les enregistrements ainsi obtenus, que les indications que j'ai données dans mon exposé sont fondées. D'importants gradients existent parfois, par temps calme, très près de la surface.

En l'absence d'autres demandes d'intervention, $M$. le Président clôt la discussion et donne la parole à M. le Professeur Woods pour l'exposé de sa communication qu'il illustre par un film en couleurs, tout à fait remarquable, pris au cours de ses expériences à la mer. 